\title{
Foreword: special issue for the journal track of the 9th Asian Conference on Machine Learning (ACML 2017)
}

\author{
Wee Sun Lee ${ }^{1}$ D $\cdot$ Robert J. Durrant ${ }^{2}$
}

Received: 26 October 2017 / Accepted: 9 November 2017 / Published online: 21 December 2017

(C) The Author(s) 2017

We welcome you to the special issue of Machine Learning, comprising of papers accepted to the journal track of the 9th Asian Conference on Machine Learning (ACML 2017), held in Yonsei University, Seoul, Korea. The ACML conference is running a dedicated journal track alongside the usual conference proceedings track for a second time. The journal track continues to be successful and we are delighted to share the contributions with you.

This year's ACML journal track received 23 submissions and six papers are accepted for this special issue. The reviewing process was handled by the ACML journal track committee, consisting of Wray Buntine from Monash University, Steven Hoi from Singapore Management University, Kee-Eung Kim from Korea Advanced Institute of Science and Technology, Hang Li from Noah's Ark Lab, Yu-Feng Li from Nanjing University, Masashi Sugiyama from RIKEN/University of Tokyo, Ichiro Takeuchi from Nagoya Institute of Technology, Ivor Tsang from University of Technology Sydney, Lijun Zhang from Nanjing University, and Jun Zhu from Tsinghua University, with co-chairs and editors of this special issue, Wee Sun Lee and Bob Durrant from National University of Singapore and University of Waikato respectively. Submitted papers were assigned to committee members by the co-chairs according to the committee members' areas of expertise while ensuring that there were no conflicts of interest. The committee members recommended reviewers and acted as meta-reviewers for the papers, with each paper receiving three or four reviews. Promising papers that did not meet the expected standard were allowed to be resubmitted after improvement, following the reviewing process of this journal.

The paper "Crowdsourcing with Unsure Option", by Yao-Xiang Ding and Zhi-Hua Zhou, analyses the problem of crowdsourcing when the workers are provided with the option of abstaining in the presence of uncertainty. Specifically, the workers are asked to abstain when

\footnotetext{
Wee Sun Lee

leews@comp.nus.edu.sg

Robert J. Durrant

bobd@waikato.ac.nz

1 National University of Singapore, Singapore, Singapore

2 University of Waikato, Hamilton, New Zealand
} 
their confidence is below some threshold $T$, assuming an incentive compatible mechanism is used to ensure that the workers behave honestly. Theoretical results giving sufficient conditions for the effectiveness of the method is provided, together with an online mechanism for selecting the threshold when properties of the crowd is not known in advance.

The paper "Robust Plackett-Luce Model for $k$-ary Crowdsourced Preferences", by Bo Han, Yuangang Pan, and Ivor W. Tsang studies the problem of aggregrating the ranking of $k$-ary preferences. Robust performance in this task has become more important with the prevalence of crowdsourcing as a method for obtaining preferences, as crowdsourced ranking data is often noisy and inconsistent. The paper proposes a method for integrating the Plackett-Luce model with a denoising vector for handling the noisy data. An online Bayesian inference method is proposed to make the method scalable, with good results shown in experiments.

In the paper "Efficient Preconditioning for Noisy Separable NMFs by Successive Projection Based Low-Rank Approximations", by Tomohiko Mizutani and Mirai Tanaka, non-negative matrix factorization under a separability assumption is studied. Non-negative matrix factorization is a computationally difficult problem in general, but becomes tractable under a separability assumption which is realistic for various practical problems. Preconditioning can be used to provide robustness against noise, and the paper provides a new computationally efficient preconditioning method. An empirical study demonstrates that the method is effective.

The paper "Semi-Supervised AUC Optimization based on Positive-Unlabeled Learning", by Tomoya Sakai, Gang Niu, and Masashi Sugiyama provides a semi-supervised learning method for maximizing the area under the receiver operation characteristic curve (AUC). Unlike typical semi-supervised learning method, the proposed method does not depend on strong distributional assumptions. The method is developed from analysis of AUC learning with positive and unlabeled data and extended to the semi-supervised case. Practical usefulness of the method is demonstrated through experiments.

In the paper "Learning Safe Multi-Label Prediction for Weakly Labeled Data", by Tong Wei, Lan-Zhe Guo, Yu-Feng Li and Wei Gao, a safe method for generating labels for incomplete training examples is proposed. The method can be used for tasks such as semi-supervised learning, where some training examples are unlabeled, as well as for weakly labeled multilabel learning, where the only parts of the labels of some of the multi-labeled examples are available. The method assumes that the ground truth labels are generated by a convex combination of base multi-label learners, and provides a labeling that maximizes the minimum of the performance measure over labelings provided by possible ground truth labelings. The $F_{1}$ score and top- $k$ precision performance measures are studied. A cutting-plane strategy is used to iteratively generate the label assignments in an efficient manner and experiments demonstrate the effectiveness of the method.

In the paper "Distributed Multi-task Classification: A Decentralized Online Learning Approach", by Chi Zhang, Peilin Zhao, Shuji Hao, Yeng Chai Soh, Bu Sung Lee, and Steven C.H. Hoi, multi-task learning over a distributed network is studied. An online two-phase decentralized learning algorithm is proposed: in the first phase, information for all tasks are shared within each node in the network, and in the second phase, communication between nodes leads to the convergence to a common multi-task minimizer. Analysis shows that the algorithm achieves a $O(\sqrt{T})$ regret bound where $T$ is the number of online iterations. Good performance is demonstrated in experiments.

We would like to thank the journal track committee for their efforts in making the special issue possible, the referees for their time and effort in reviewing the papers and the authors for their contributions. We also wish to thank Peter Flach, editor-in-chief for MLJ, Dragos Margineantu, editor of special issues for MLJ, as well as the ACML Steering Committee for 
their guidance and support. Thanks as well to Melissa Fearon from the Springer editorial office for the help in ensuring that the process ran smoothly.

The papers in this special issue contain many interesting ideas. We hope that you enjoy them and find the papers useful for your research. 